\title{
The Irregularity Propagation Characteristics of Radio Signals For Wireless Sensor Network In Farmland
}

\author{
Hua-Ji ZHU ${ }^{1,2,3}$, Hua-Rui WU ${ }^{1,2,3, *}$, Li-Hong ZHANG ${ }^{1,2,3}$ and Yi-Sheng MIAO ${ }^{1,2,3}$ \\ ${ }^{1}$ National Engineering Research Center for Information Technology in Agriculture, Beijing 100097, PR China \\ ${ }^{2}$ Beijing Research Center for Information Technology in Agriculture, Beijing Academy of Agriculture and Forestry Sciences, Beijing 100097, PR \\ China \\ ${ }^{3}$ Key Laboratory for Information Technologies in Agriculture, Ministry of Agriculture, Beijing 100097, PR China \\ zhuhj@nercita.org.cn,wuhr@nercita.org.cn, zhanglh@nercita.org.cn,miaoys@nercita.org.cn \\ ${ }^{*}$ Corresponding author: Hua-Rui WU
}

\begin{abstract}
This work aims to investigate the irregular propagation characteristics of wireless sensor network (WSN) at frequency of $433 \mathrm{MHz}$. Through the analysis of the received signal strength indicator (RSSI), it is found that the variance in received signal strength is largely random, along with a continuous change with incremental changes in direction. For the transceiver distance is $20 \mathrm{~m}$, the packet loss rate (PLR) in all directions is relatively small except for the east direction, indicating that the signal is still strong in all directions. While for the transceiver distance is $40 \mathrm{~m}$, it can be found that there is about $90 \%$ packet loss in the east direction. That is, the received signal strength in the east direction is lower than that in the other directions. Moreover, the communication range varies with the degree of receiver direction ranging from 0 to 359. Through the regression analysis in Matlab, we find that the optimal fitting models in different directions are different. The optimal fitting model in east and west direction is the modified exponential decay, and in south and north direction is the linear logarithmic model. The values of $\mathrm{R}^{2}$ vary from 0.935 to 0.961 , and the values of RMSE range from 1.75 to 2.31 .
\end{abstract}

\section{Introduction}

Wireless sensor network (WSN) is a recent novel technology and it has been increasingly used in agriculture [1]. WSN is composed of a number of sensor nodes which are capable of sensing, processing, and transmitting environmental information [2]. The sensor nodes are communicate with each other through wireless radio signals $[3,4]$.

In recent years, many studies have been performed to study the characteristics of wireless radio propagation through measuring the antenna height, vegetation depth, and the propagation frequency. Suwalak et al. [5] studied the propagation characteristics of a single vegetation type, such as durian and plum. Moreover, Azevedo et al. [6] researched the propagation characteristics of wireless radio in some complex agricultural environments such as forests with dozens of tree species. Ndzi et al. [7] conducted the wireless sensor network coverage measurements in a mixed crop farmland, and proposed a log-linear model. The existing empirical model regard the propagation environment as isotropic homogeneous. Unfortunately, radio irregularity is a common and non-negligible phenomenon in wireless sensor networks [8].

In order to characterize irregular propagation for WSN in agricultural fields like wheat filed, RF signal propagation experiments at different directions are done at frequency of $433 \mathrm{MHz}$. In this study, we investigate the impact of transceiver distance and degree of receiver direction on the propagation of RF signal. First, we study the received signal strength indicator (RSSI) and packet loss rate (PLR) at various transceiver distances at different directions. Then, we obtain the communication range at different directions. Finally, we focus on the regression analysis of path loss at various directions.

\section{Related work}

The radio irregularity can result in irregularity in radio range and variations in packet loss in different directions [9]. Ganesan et al. [10] found that the radio range varies significantly in different directions. The radio irregularity is mainly due to the variance of the signal path loss. Shankar et al. [11] proposed that the wireless radio signal may be reflected, diffracted, and scattered for propagating within a 
medium. Generally, the medium is different in different directions. Hence, radio propagation presents anisotropic in most environments.

\section{Experimental method}

As a major food crops in Northern China, winter wheat is taken as the research object in this paper. The impact of plant on RF signal propagation characteristic in different growing stages is different. Here, we choose the elongation stage for testing, and the average plant height is $0.40 \mathrm{~m}$. We carry out the experiment at the wheat-field in xiaotangshan national precision agriculture research demonstration base. The 1100 SE RF wireless sensor node is selected, and the devices are equipped with the frequency of $433 \mathrm{MHz}$. The transmitted power and the Omni-directional antenna gain are $0 \mathrm{dBm}$, and $4 \mathrm{~dB}$, respectively. To avoid unnecessary loss caused by antenna polarity, transmitter and receiver are at the same height and kept in the same direction. The receiver sensitivity is $-110 \mathrm{dBm}$. The RSSI and PLR are obtained for antenna heights of transmitter and receiver are $0 \mathrm{~m}$.

\section{Experimental results}

\subsection{Anisotropic RSSI}

In this study, it is supposed that the antenna height of transmitter $\left(h_{t}\right)$ and receiver $\left(h_{r}\right)$ are the same, i.e., $h_{t}=h_{r}=$ $\mathrm{h}$ [12]. As shown in Figure 1, the RSSI at $433 \mathrm{MHz}$ is plotted as a function of the number of measurement at different directions. It is found that received signal strength in each direction is relatively stable, and the small variance can be attributed to the fading effect [11]. Despite the same distance between transceiver, the received signal strength in the south is much higher than that in the east. Moreover, we study the relation between the signal strength and the degree of angular direction for the transceiver distance is 20 $\mathrm{m}$ and $40 \mathrm{~m}$, respectively. As shown in Figure 2, the received signal strength varies continuously with the change of direction. That is to say, the incremental changes in direction lead to the incremental variation for RSSI, which is agreement with previous works [13].

\subsection{Anisotropic PLR}

The relationship between PLR and the degree in directions for the transceiver distance of $20 \mathrm{~m}$ and $40 \mathrm{~m}$ is presented in Figure 3. As shown in Figure 3, the PLR for the transceiver distance of $20 \mathrm{~m}$ is relatively small in all directions, except for in the east direction. In other words, the signal is still strong in other three directions. While for the transceiver distance is $20 \mathrm{~m}$, it can be seen that there is about $90 \%$ packet loss in the east direction. The phenomenon demonstrates that the received signal strength in the east direction is lower than that in the other directions, which is consistent with the results in Figure 1. Interestingly, the PLR in north direction is relatively small in two transceiver distance in comparison with the other three directions.

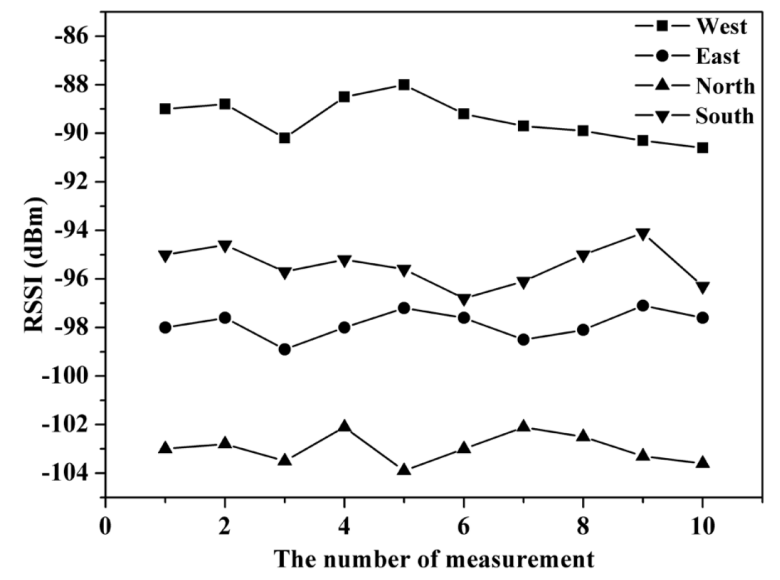

Figure 1. The Rssi of Various Number Of Measurement In Four Directions.
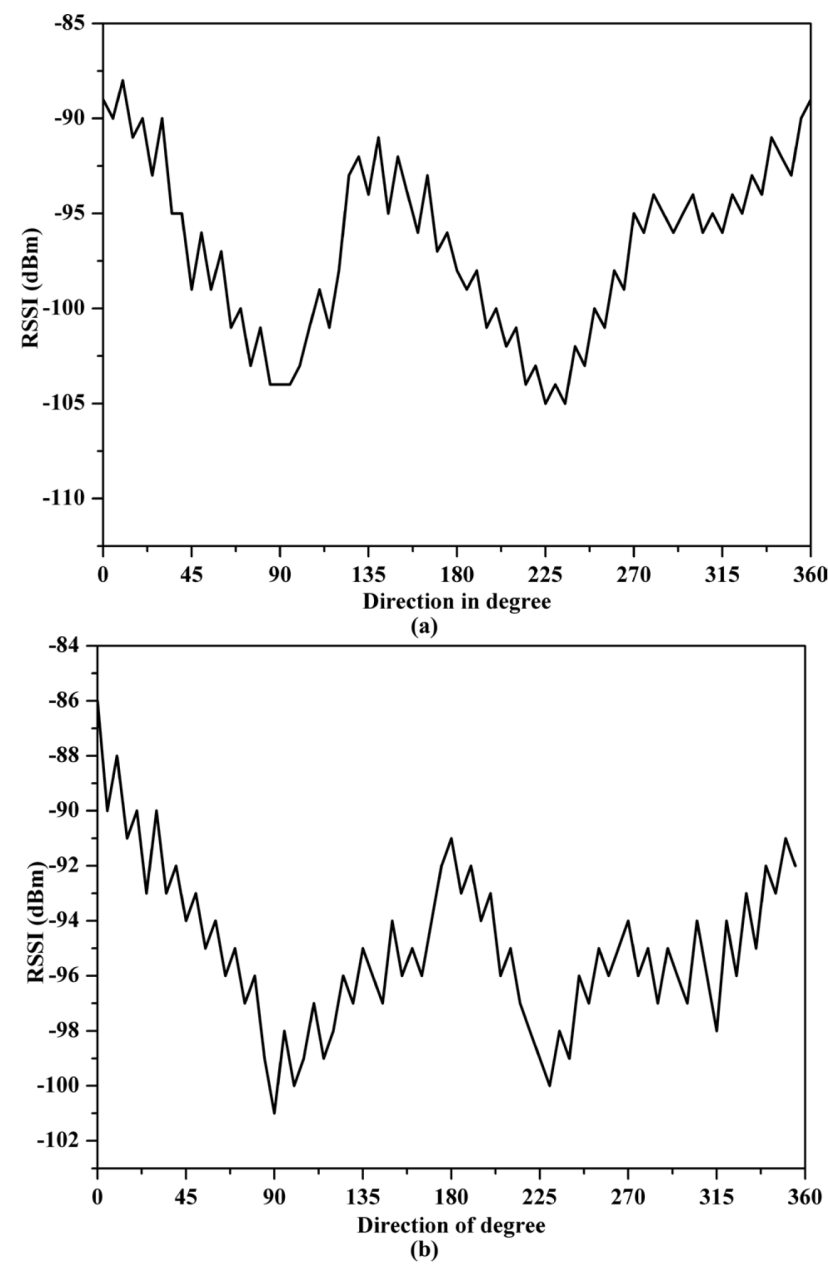

Figure 2. The rssi in Different Degree in Four Directions. (a) the Transceiver Distance is $20 \mathrm{~m}$ (b) the Transceiver Distance is $40 \mathrm{~m}$. 


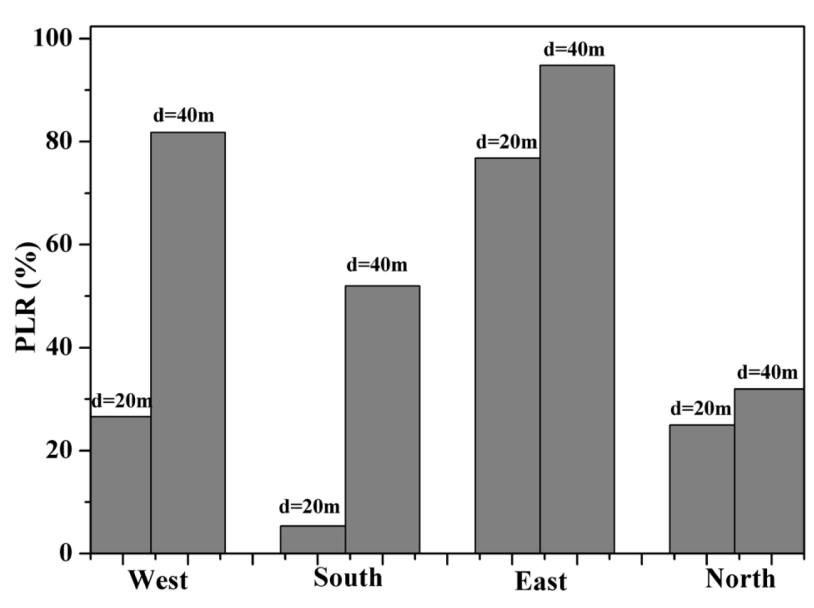

Figure 3. The Plr for Transceiver Distance is $20 \mathrm{~m}$ and $40 \mathrm{~m}$ in Four Directions.

\subsection{Anisotropic communication range}

To further demonstrate the irregularity, we research the communication range in all directions. In the experiment, the receiver sensitivity is $-110 \mathrm{dBm}$. Then with such thresholds, the communications in different directions are measured. As shown in Figure 4, the communication range varies with the degree of receiver direction ranging from 0 to 359 . In other words, the communication range is not uniform in all directions. The range map may be another illustrations for radio irregularity in a wireless medium.

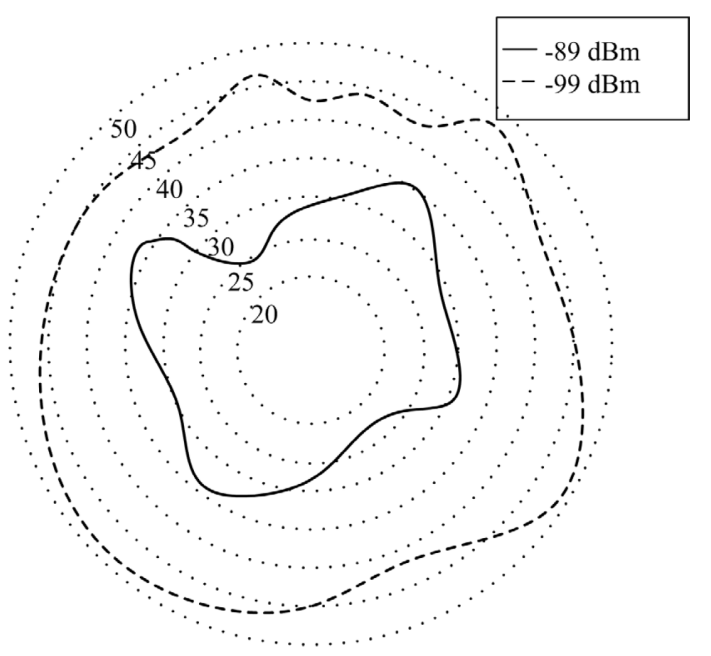

Figure 4. The Anisotropic Communication Range of Different Degree in Direction.

\subsection{The regression analysis for path loss}

For wireless signal propagate between transmitter and receiver, radio waves interact with environment, causing path loss [14]. Path loss (PL) is defined as the difference between the transmitted and the received power. It can be expressed by:

$$
P L=P_{t}+G_{t}+G_{r}-P_{r}(d)
$$

Where $P_{t}$ is the transmitted power, $G_{t}$ is the antenna gain of transmitter, $G_{r}$ is the antenna gain of receiver, and $P_{r}(d)$ is the received power. In a transmission medium, the received power can be expressed by:

$$
P_{r}=P_{t} G_{t} G_{r}(\lambda / 4 \pi d)^{n}
$$

Where $\lambda$ and $d$ are the wavelength of the wireless signal, and the distance between the transmitter and receiver, respectively. $\mathrm{n}$ is the transmission factor which value depends on the environment of propagation. For wireless signal propagation in free space, we assume that the wireless signal travels in a homogeneous medium. Under this circumstance, the rate of attenuation is constant, i.e., $n$ is equal to 2 [15]. Generally speaking, the power attenuation can be defined as follows:

$$
P L=10 \log _{10}\left(P_{t} / P_{r}\right)
$$

Furthermore, the path loss in free space can be simplified as:

$$
P L_{\text {Freespace }}=20 \log _{10}(f)+20 \log _{10}(d)-27.56
$$

Where $\mathrm{f}$ is the frequency of RF signal. Through the logarithm processing, the equation (3) can be converted into the equation as follows:

$$
P L=A-10 * n * \log _{10}(d)
$$

According to the previous studies $[15,16]$, there are many vegetation attenuation models, such as Non-zero Gradient model and Modified Exponential Decay (MED) model. From a propagation mechanisms perspective, at very shallow vegetation depths, signal propagation is dominated by coherent components which decrease rapidly with distance [15]. Beyond this point, propagation is mainly due to forward scattering and the signal is dominated by diffuse components [15]. However, because of the small transmitted power from wireless sensor nodes, most scattered or diffused components are very weak. Nonetheless, their presence is illustrated by the reduction in the signal decay gradient at distances further from the transmitter [16]. The generalized form of the MED vegetation attenuation models is given by:

$$
P_{r}=X f^{Y} d^{Z}
$$

Where $\mathrm{f}$ and $\mathrm{d}$ are the frequency in megahertz $(\mathrm{MHz})$ and the vegetation depth in meters. Moreover, $\mathrm{X}, \mathrm{Y}$, and $\mathrm{Z}$ are model parameters.

Based on the above models, we make regression analysis for path loss at different directions in Matlab. As 
we know, the important indicator in curve fitting is $\mathrm{R}^{2}$ which reflect the correlation between theoretical calculation values and measured values. Generally speaking, $\mathrm{R}^{2}$ is more closer to one, the correlation between theoretical calculation values and measured values is greater. To evaluate the reliability of models application, Root Mean Square Error (RMSE) between the models and the measured data are further calculated. The RMSE is a frequently used measure of the difference between values predicted by a model and the values actually measured from the environment [17]. RMSE is a good measure for accuracy of the models, and can be expressed by:

$R M S E=\sqrt{\frac{\sum_{i=1}^{n}\left(X_{\mathrm{obs}, i}-X_{\mathrm{mod}, i}\right)}{n}}$

Where $X_{\text {obs }}, X_{\text {mod, }}$, and $n$ are the measured values, the modeled values, and the number of samples, respectively. The fitting curves of path loss in four directions for the frequency of $433 \mathrm{MHz}$ are presented in Figure 5. It can be observed that the differences of path loss in different directions are obvious. At frequency of $433 \mathrm{MHz}$, the path loss in east direction can be expressed by:

$$
P L=0.627 * f^{0.712} * d^{0.098}
$$

While for the west direction, the path loss can be expressed by:

$$
P L=0.084 * f^{0.816} * d^{0.331}
$$

In the south direction, the path loss model can be presented by:

$$
P L=41.83+25.8 * \log _{10}(d)
$$

In the north direction, the path loss model can be expressed by:

$$
P L=38.54+36.4 * \log _{10}(d)
$$

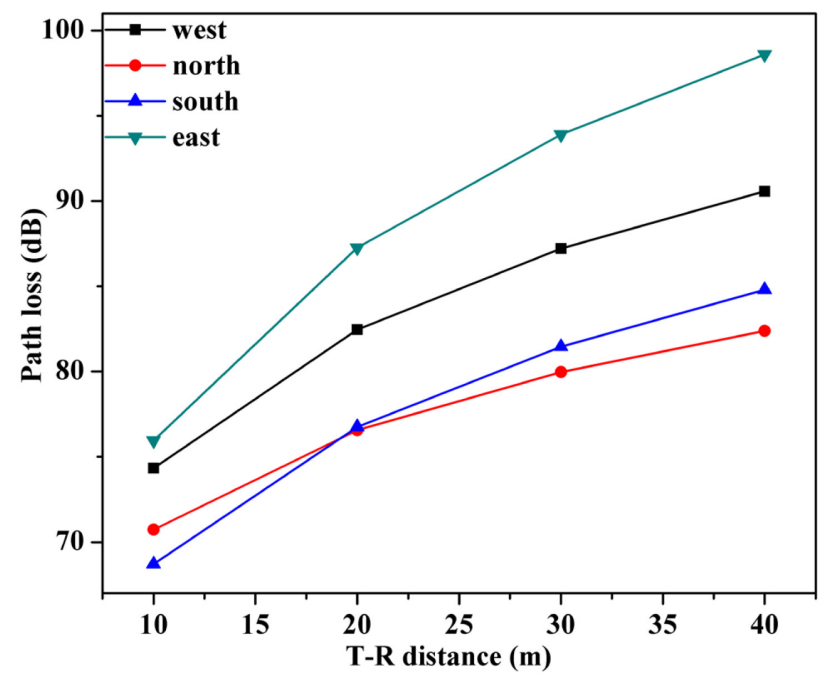

Figure 5. The Measured and the Fitting Path Loss in Four Different Directions.

\section{Conclusions}

This work aim to study the irregular propagation characteristics of wireless sensor network at frequency of $433 \mathrm{MHz}$. The radio irregularity depend on the direction of propagation, and can be caused by multiple factors, such as variance in RF sending power and different path losses. Through the analysis of the received signal strength indicator, it can be found that the variance in received signal strength is largely random, along with a continuous change with incremental changes in direction. For the transceiver distance is $20 \mathrm{~m}$, the packet loss rate in all directions is relatively small except for the east direction, indicating that the signal is still strong in all directions. While for the distance is $40 \mathrm{~m}$, it can be found that there is about $90 \%$ packet loss in the east direction. That is, the received signal strength in the east direction is lower than that in the other directions. Moreover, the communication range varies with the degree of receiver direction ranging from 0 to 359. Through the regression analysis in Matlab, we find that the optimal fitting models in different directions are different. The optimal fitting model in east and west direction is the modified exponential decay, and in south and north direction is the linear logarithmic model. The values of $\mathrm{R}^{2}$ vary from 0.935 to 0.961 , and the values of RMSE range from 1.75 to 2.31 at frequency of $433 \mathrm{MHz}$.

\section{Acknowledgments}

This work is supported by Natural Science Foundation of China (61571051, 61471067, 61271257). 


\section{References}

1. Kone C. T., Hafid A, Boushaba M, IEEE. Sens. J, 15, $10(2015)$

2. An W, Ci S, Luo HY, Wu DL, Adamchuk V, Sharif H, Wang XY, Tang H, Wireless Communications and Mobile Computing, 15, 12 (2015)

3. Liu WM, Rahman M. F, Thirumuruganathan S, Nan Z, Das G, Proceedings of the VLDB Endowment, 8, 12 (2015)

4. Mao DD, Sun WW, Chen CN, Wu JJ, Liu WM, Jing YN, Journal of Chinese Computer Systems, 32, 6 (2011)

5. Vougioukas S, Anastassiu H. T, Regen C, Zude M, Biosyst Eng, 114, 4 (2013)

6. Azevedo J. A. R, Santos F. E. S, IEEE T Anten Propag, 59, $6(2011)$

7. Ndzi D. L, Harun A, Ramli F. M, Kamarudin M. L, Zakaria A, Shakaff A. Y. M, Jaafar M. N, Zhou Shikun, Farook R. S, Comput Electron Agr, 105 (2014)

8. Guo XM, Zhao CJ, Int J. Agr. Biol. Eng, 7, 6 (2014)

9. Cerpa A, Busek N, Estrin D, Center for Embedded Network Sensing, (2003)

10. Ganesan D, Krishnamachari B, Woo A, Culler D, Wicker S, Technical Report UCLA/CSD-TR 02, (2002)

11. Hamid M. A, Abdullah-Al-Wadud M, Chong I, The International Conference on Information Networking 2011 (ICOIN2011). IEEE, (2011)

12. Sai S, Niwa E, Mase K, Nishibori M, Inoue J, Obuchi M, Kizu M, IEEE Commun Mag, 47, 11 (2009)

13. Zhou G, He T, Krishnamurthy S, Stankovic J. A, ACM T Sensor Network, 2, 2 (2006)

14. Harun A, Shakaff A. Y. M, Zakaria A, Kamarudin L. M, Jaafar M. N., Ndzi D. L., 2011 Third International Conference on Computational Intelligence, Modelling \& Simulation. IEEE, (2011)

15. Zhou, G., He, T., Krishnamurthy, S., Stankovic, J. A, In Proceedings of the 2nd international conference on Mobile systems, applications, and services, (2004)

16. Meng Y S, Lee Y H, Investigations of foliage effect on modern wireless communication systems: A review[J]. Prog Electromagn Res, 105 (2010)

17. Ndzi D. L., Kamarudin L. M., Muhammad Ezanuddin A. A., Zakaria A, Ahmad R. B., Malek M. F. B. A., Jafaar M. N, Prog Electromagn Res B, 36 (2012) 